\title{
Access to child care services in the Northeast and in the South Regions of Brazil
}

Andréia Soprani dos Santos 1

Suele Manjourany Siva Duro 2

Nagela Valadão Cade 3

Luiz Augusto Fachini 4

Elaine Tomasi 5

\footnotetext{
Departamento de Ciências da Saúde. Universidade Federal do Espírito Santo. São Mateus, ES, Brasil. CEP: 29.932-540. E-mail: andreia soprani@hotmail.com

2 Faculdade de Enfermagem. Universidade Federal de Pelotas. Pelotas, RS, Brasil.

3 Centro de Ciências da Saúde. Universidade Federal do Espírito Santo. Vitória, ES, Brasil.

4,5 Departamento de Medicina Social. Universidade Federal de Pelotas. Pelotas, RS, Brasil.
}

\begin{abstract}
Objectives: to estimate the incomplete follow-up on child care services and the associated factors in the municipalities in the Northeast and in the South of Brazil.

Methods: a population-based cross-sectional study with 7,915 children. The incomplete follow-up on child care regarded the absence of at least one of the seven advocated care services. The Poisson regression was used for crude and adjusted analysis.

Results: the prevalence of the incomplete follow-up child care was $53.6 \%(C I 95 \%=52.5$ $54.7)$ in the Northeast and $28.3 \%(C 195 \%=27.3-29.3)$ in the South, therefore $91 \%$ was greater in the Northeast $(P R=1.91 ; C 195 \%=1.73-2.11)$. Protector effect was observed: in the muni-cipalities with 30 to 49 thousand inhabitants in the Northeast $(P R=0.72 ; C 195 \%=$ 0.64-0.82) and 100 to 999 thousand inhabitants in the South (PR=0.69; CI95\%=0.57-0.84); the South has the greatest income quartile $(R P=0.77 ; C 195 \%=0.63-0.95)$ and has six or more prenatal consultations ( $P R=0.83 ; C 195 \%=0.75-0.92)$ in the Northeast and $(P R=0.65$; $C I 95 \%=0.53-0.79)$ in the South. Social classes D and E showed risks (PR=1.41; CI95\%= 1.19-1.67) in the Northeast and $(P R=1.67 ; C 195 \%=1.37-2.03)$ in the South.

Conclusions: children in the Northeast are more likely not to have a complete child care follow-up, implying that the user does not come to be attended or finds difficulties to have access to the health services.
\end{abstract}

Key words Health services accessibility, Health inequalities, Cross-sectional studies, Child health 


\section{Introduction}

The access to health services can be understood as an easy way to obtain assistance that may give opportunities in receiving better health results, $1,2,3$ which entails the removal of obstacles to reach the offered services. ${ }^{4}$ Thus, the use of a certain service can be considered as a dimension to the achieved access $^{5}$ while its absence entails the lack of access.

In Brazil, although the Federal Constitution guarantees complete health access and integrality assistance in an evenly way for all citizens, ${ }^{6,7}$ which there are still extensive disparities. Socioeconomic and regional differences, along with the individual's characteristics have been identified as influential in restricting access to the health services. $8-10$ The strategies for this understanding remains to be the aim of profound debates given by the complexity of factors that surrounds it and the necessity of organizational analysis in the health system.2,4

One of the assumptions in explaining the restriction of access constitutes the organization and the health services. ${ }^{8}$ Poorer regions have an unfavorable context regarding the organization and the services offered, $6,10,11$ while the population with a better socioeconomic level have access to more health services.

In relation to child health services, and especially in child care, it is fundamentally understood that the early health care service should start at least with seven routine visits in the child's first year of life and it should occur preferably in the 1 st week and in the 1 st $, 2^{\text {nd }}, 4^{\text {th }}, 6^{\text {th }}$, 9th and $12^{\text {th }}$ months, 12 and be able to ensure the child's health surveillance. The continuous child care service contributes to neuropsychomotor development and follow-up, stimuli for breastfeeding and the introduction of new food, weight control, immune-preventable diseases and the production of healthy habits, as though health support and prevention on various diseases in the child's first years of life. 12 The development of actions related to these goals are attributed to all the basic health units in Brazil.12 Data point out that in the Northeast and in the South regions, child care is present in almost $100 \%$ of the Family Health Strategy (ESF) units. ${ }^{13}$ Considering the significant advance of the ESF expansion in the country, $6,14,15$ the full access to the health service for all the children can and should be guaranteed in the equity pursuit in health. Although the country has made important progress for the population of children, such as the reduction of mortality, adequate levels of vaccine coverage 6 and an average increase in the prevalence of breastfeeding, 7 however, there are still gaps in the equity access.

In order to contribute in understanding some barriers to have access to the health services, this study aimed to estimate the incomplete follow-up on child care and the associated factors in the municipalities in the Northeast and in the South of Brazil.

\section{Methods}

This study had a population-based cross-sectional design and was carried out in urban census sectors in 35 municipalities in the Northeast and in the South Regions of Brazil. The homes where the children under the age of seven and their families lived were located in areas with traditional Basic Health Units (UBS) and ESF. This study integrates with the research on "Situação de saúde, utilização de serviços e qualidade da atenção em crianças e familiares nas Regiões Sul e Nordeste do Brasil"16 (Health situation, the use of services and quality care in children and their families in the South and in the Northeast regions of Brazil") carried out in August to October 2010.

A sample survey was performed for the main research, considering in the regions the municipalities with ESF coverage of $30 \%$ to $70 \%$. The municipalities were randomly selected based on a stratification of four population sizes: 10 thousand to less than 29 thousand inhabitants; 30 thousand to less than 50 thousand; 50 thousand to less than 100 thousand; and 100 thousand to less than 1 million inhabitants. A population percentage distribution of each size of the municipalities was calculated and this distribution was considered for the selection of the sample. The whole sampling procedure is available in Facchini et al. ${ }^{16}$ study

The basic care services from each chosen municipality were organized through lists with the identification of the address, the model of care and the census sectors in its coverage area. For each included service, two census sectors were selected, one that included the health service and the other closest to the health unit that composed with the sample.

In each sector was chosen at a starting point to locate the first household of the study and the sample of children. In the sequence, the remaining households were included considering the interval of five households from the starting point. In each household, all the children under the age of seven were eligible for this study

For this study, the sample was restricted to children between one and four years old, aiming to know the complete exposure to child care at the end of the 
child's first year of life and to minimize possible biases related to the older children's mothers' memory. The sample calculation was performed a posteriori, establishing level of significance at $95 \%$, power at $80 \%$, prevalence at $34 \%$ for the lack of access to child care and the prevalence ratio of 1.2. Also considering, $10 \%$ for losses or refusals and $30 \%$ for controlling confounding factors which resulted in 6,163 children.

Data collection was performed by previously trained interviewers with the help of a personal digital assistant (PDA). An individual questionnaire containing information about each child and his / her mothers and a socioeconomic questionnaire with information about the household members were applied. The questionnaires were answered at home interviews by the birth mother or, in her absence, by a responsible resident. One of the quality control actions was the re-interview of $8 \%$ of the sample with the repetition of four information about the child and family. The concordance analysis revealed Kappa indices between 0.6 and 0.9 .

The prevalence of each child care services performed at fifteen days, one, two, four, six, nine and twelve months of age was analyzed separately. These variables were then used to construct a complex outcome, considering as an incomplete follow-up on child care if the child was not attended in at least one of the seven proposed visits.

The independent variables were grouped into five categories: geographic location - Region (South and Northeast) and the municipal size in one thousand inhabitants ( 10 to 29,30 to 49,50 to 99 and 100 to 999); socioeconomic level - economic classification according to Associação Brasileira de Empresas de Pesquisas (The Brazilian Association Research Companies) (ABEP)17 (A/B, C, D/E) expresses the household wealth and the power to purchase including, for example, household goods and the family provider's schooling level, therefore, A is the richest level, the family income per capita in minimum wage quartiles (less than $0.237 ; 0.238$ to $0.431 ; 0.432$ to 0.823 ; and 0.824 or more) and Family Welfare (Bolsa Família) program (yes or no); maternal demographic and social issues - age in years (19 or less, 20 to 29,30 to 39 and 40 or more), self-referred skin color (white, mixed, black, oriental, and native Brazilian), schooling in complete years (4 or less, 5 to 8, and 9 or more), presence of a partner (yes or no); obstetrical history - total number of prenatal consultations (up to 5 and 6 or more) and number of live births (1, 2, 3 or more); the child's characteristics - sex (male or female), age in years $(1,2,3,4)$ and the mother's self referred skin color (white, mixed, black, oriental and native Brazilian).

Bivariate analyzes were performed among all independent variables with prevalence calculation and the significance of the associations was assessed with the chi-square test on heterogeneity and linear tendency. For the synthetic outcome analysis, the Poisson regression with robust variance adjustment was used to estimate the prevalence ratios (PR) with their respective $95 \%$ confidence intervals (CI95\%). In the adjusted analysis, the hierarchical model was used, in which the geographic location variables were included in the first level; in the second level, the socioeconomic ones; in the third level, those related to maternal demographic and social characteristics; in the fourth level, the obstetric history; and, finally, in the fifth level, the variables related to the characteristics of the child. For the entry and maintenance of the variables in the model presenting value $p<0.20$ were considered. The statistical significance was verified by Wald test and the heterogeneity adopting $5 \%$ level. All the data analysis was conducted in the Stata 13.0 statistical package.

The research project was approved by the Ethics Committee at Faculdade de Medicina da Universidade Federal de Pelotas, according to the document number 133/09, on December 21st, 2009. The Informed Consent Form was signed by all the interviewees.

\section{Results}

In a total of 7,915 children aged between one and four years old in the States of Rio Grande do Sul $(\mathrm{n}=715), \quad$ Santa Catarina $\quad(\mathrm{n}=1,997), \quad$ Paraná $(\mathrm{n}=1,182)$, Bahia $(\mathrm{n}=1,777)$, Pernambuco $(\mathrm{n}=1,506)$ and Ceará $(\mathrm{n}=758)$ integrated this sample.

A little over half of the children lived in the Northeast $(51.1 \%)$, most of them in municipalities with more than 100 thousand inhabitants $(59.7 \%)$, belonged to families in an economic classification $\mathrm{C}$ (53.0\%), did not receive the Family Welfare (Bolsa Família) program (64.9\%), and half of them lived on with less than 0.43 monthly minimum wage per capita. Most of the mothers were under 30 years old, self referred as mixed skin color $(48.1 \%)$, attained high school $(54.1 \%)$, had a partner $(78.8 \%)$, had more than five prenatal consultations $(85.7 \%)$ and were primiparous $(43.8 \%)$. Among the children, slightly more than half of them were male, less than two years old $(50.8 \%)$ and were white $(52.2 \%)$ (Table 1).

Tables 2 and 3 demonstrate the incomplete follow-up on child care in the Northeast and in the 
South Regions, respectively. In the Northeast, the prevalence of this outcome reached values close to $30 \%$ along the seven visits. In the South, the prevalence was considerably lower, did not reach $10 \%$ in most of the visits. The economic, schooling and health service characteristics exerted the greatest influence in the results in both regions. On the other hand, in both regions, maternal (age, color and presence of partner) and child variables were not related to the incomplete follow-up on child care.

Among the reasons mentioned by the mothers for not taking their children to the health unit for consultations during their first year of life, $60 \%$ of the mothers reported saying that the community health agents weighed and measured the children at home (data not shown)

Considering the complex outcome, the proportion of children who had an incomplete follow-up on child care in the Northeast region was almost the double $(53.6 \%$, CI95\% $=52.5-54.7)$ comparing to the South Region (28.3\%, CI95\% $=27.3-29.3)$ for all seven visits. In the adjusted analysis according to the hierarchical model, the Northeast presented a risk of 1.91 $(\mathrm{PR}=1.91 ; \mathrm{CI} 95 \%=1.73-2.11)$ and the South showed a protective effect for the incomplete follow-up on child care $(\mathrm{PR}=0.54, \mathrm{CI} 95 \%=0.49-0.59)$, for both regions, the factors that were associated to the outcome were: the size of the municipality with a difference in the effect for the Northeast $(\mathrm{PR}=1.22$; $\mathrm{CI} 95 \%=1.08-1.39$ ); population of 50 to 99 thousand inhabitants, compared to the South $(\mathrm{PR}=0.69$, $\mathrm{CI} 95 \%=0.57-0.84)$; more than 100 thousand inhabitants; economic classification D and E, thus, a little more expressive in the South $(\mathrm{PR}=1.67$, $\mathrm{CI} 95 \%=1.37-2.03)$ than in the Northeast $(\mathrm{PR}=1.41$, $\mathrm{CI} 95 \%=1.19-1.67)$ and the use of prenatal services, maintaining the same effect for the Northeast $(\mathrm{PR}=0.83$, CI95\% $=0.75-0.92)$ and for the South $(\mathrm{PR}=0.65, \mathrm{CI} 95 \%=0.53-0.79)$. The variables: maternal schooling and the presence of the partner when submitted to the adjusted analysis were not associated to the outcome. (Table 4).

\section{Discussion}

Altogether, more than half of the children in the Northeast Region and just a little over a quarter of the children in the South Region did not receive the complete follow-up on child care. The risk effect

Table 1

Sample description according to demographic, socioeconomic, maternal and children's variables in the Northeast and the South Region. Brazil, 2010.

\begin{tabular}{|c|c|c|c|c|c|c|}
\hline \multirow[t]{3}{*}{ Variables } & \multicolumn{4}{|c|}{ Region } & \multicolumn{2}{|c|}{ Total } \\
\hline & \multicolumn{2}{|c|}{ Northeast } & \multicolumn{2}{|c|}{ South } & \multirow[b]{2}{*}{$\mathrm{n}$} & \multirow[b]{2}{*}{$\%$} \\
\hline & $\mathrm{n}$ & $\%$ & $\mathrm{n}$ & $\%$ & & \\
\hline \multicolumn{7}{|c|}{ Municipal size in thousands inhabitants } \\
\hline 10 to 29 & 333 & 8.2 & 288 & 7.4 & 621 & 7.9 \\
\hline 30 to 49 & 829 & 20.5 & 736 & 19.0 & 1,565 & 19.7 \\
\hline 50 to 99 & 258 & 6.4 & 746 & 19.3 & 1,004 & 12.7 \\
\hline 100 to 999 & 2,621 & 64.9 & 2,104 & 54.3 & 4,725 & 59.7 \\
\hline \multicolumn{7}{|c|}{ Economic classification } \\
\hline$A$ and $B$ & 304 & 8.1 & 1,271 & 34.5 & 1,575 & 21.1 \\
\hline $\mathrm{C}$ & 1,917 & 50.8 & 2,034 & 55.3 & 3,951 & 53.0 \\
\hline $\mathrm{D}$ and $\mathrm{E}$ & 1,553 & 41.2 & 376 & 10.2 & 1,929 & 25.9 \\
\hline \multicolumn{7}{|c|}{ Per capita income in minimum wages quartiles } \\
\hline$<0.237$ & 1,404 & 39.0 & 363 & 10.4 & 1,767 & 24.9 \\
\hline 0.238 to 0.431 & 1,136 & 31.5 & 635 & 18.2 & 1,771 & 25.0 \\
\hline 0.432 to 0.823 & 669 & 18.6 & 1,114 & 32.0 & 1,783 & 25.2 \\
\hline 0,824 & 392 & 10.9 & 1,373 & 39.4 & 1,765 & 24.9 \\
\hline \multicolumn{7}{|c|}{ Family Welfare Program (Bolsa Família) } \\
\hline Yes & 2,007 & 50.5 & 721 & 19.0 & 2,728 & 35.1 \\
\hline No & 1,996 & 49.5 & 3,083 & 81.1 & 5,049 & 64.9 \\
\hline
\end{tabular}


Sample description according to demographic, socioeconomic, maternal and children's variables in the Northeast and the South Region. Brazil, 2010.

\begin{tabular}{|c|c|c|c|c|c|c|}
\hline \multirow[t]{3}{*}{ Variables } & \multicolumn{4}{|c|}{ Region } & \multicolumn{2}{|c|}{ Total } \\
\hline & \multicolumn{2}{|c|}{ Northeast } & \multicolumn{2}{|c|}{ South } & \multirow[b]{2}{*}{$\mathrm{n}$} & \multirow[b]{2}{*}{$\%$} \\
\hline & $\mathrm{n}$ & $\%$ & $\mathrm{n}$ & $\%$ & & \\
\hline \multicolumn{7}{|c|}{ Maternal age in years } \\
\hline 19 or less & 263 & 8.8 & 206 & 7.1 & 469 & 8.0 \\
\hline 20 to 29 & 1,676 & 56.1 & 1,422 & 49.1 & 3,098 & 52.6 \\
\hline 30 to 39 & 884 & 29.6 & 991 & 34.2 & 1,875 & 31.9 \\
\hline 40 or more & 166 & 5.5 & 275 & 9.5 & 441 & 7.5 \\
\hline \multicolumn{7}{|l|}{ Maternal skin color } \\
\hline White & 650 & 21.6 & 2,089 & 71.7 & 2,739 & 46.2 \\
\hline Mixed & 2,134 & 70.8 & 716 & 24.6 & 2,850 & 48.1 \\
\hline Black & 212 & 7.1 & 86 & 3.0 & 298 & 5.0 \\
\hline Oriental & 13 & 0.4 & 12 & 0.4 & 25 & 0.4 \\
\hline Native Brazilian & 4 & 0.1 & 9 & 0.3 & 13 & 0.3 \\
\hline \multicolumn{7}{|c|}{ Maternal schooling in years } \\
\hline 4 or less & 376 & 13.5 & 267 & 9.6 & 643 & 11.5 \\
\hline 5 to 8 & 962 & 34.5 & 957 & 34.3 & 1,919 & 34.4 \\
\hline 9 or more & 1,454 & 52.1 & 1,566 & 56.1 & 3,020 & 54.1 \\
\hline \multicolumn{7}{|l|}{ Presence of partner } \\
\hline Yes & 2,241 & 75.1 & 2,392 & 82.6 & 4,633 & 78.8 \\
\hline No & 743 & 24.9 & 504 & 17.4 & 1,247 & 21.2 \\
\hline \multicolumn{7}{|c|}{ Number of prenatal consultations } \\
\hline Up to 5 & 655 & 18.3 & 361 & 10.3 & 1,016 & 14.3 \\
\hline 6 or more & 2,932 & 81.7 & 3,154 & 89.7 & 6,086 & 85.7 \\
\hline \multicolumn{7}{|c|}{ Number of live births } \\
\hline 1 & 1,331 & 44.5 & 1,247 & 43.0 & 2,578 & 43.8 \\
\hline 2 & 964 & 32.2 & 960 & 33.1 & 1,924 & 32.6 \\
\hline 3 or more & 699 & 23.4 & 692 & 23.9 & 1,391 & 23.6 \\
\hline \multicolumn{7}{|l|}{ Child's sex } \\
\hline Male & 2,077 & 51.4 & 2,037 & 52.6 & 4,114 & 52.0 \\
\hline Female & 1,964 & 48.6 & 1,837 & 47.4 & 3,801 & 48.0 \\
\hline \multicolumn{7}{|l|}{ Child's age in years } \\
\hline 1 & 1,016 & 25.1 & 1,054 & 27.2 & 2,070 & 26.2 \\
\hline 2 & 1,069 & 26.5 & 899 & 23.2 & 1,968 & 24.8 \\
\hline 3 & 990 & 24.5 & 953 & 24.6 & 1,943 & 24.6 \\
\hline 4 & 966 & 23.9 & 968 & 25.0 & 1,934 & 24.4 \\
\hline \multicolumn{7}{|l|}{ Child's skin color } \\
\hline White & 1,204 & 29.8 & 2,919 & 75.3 & 4,123 & 52.2 \\
\hline Mixed & 2,653 & 65.7 & 854 & 22.1 & 3,507 & 44.4 \\
\hline Black & 148 & 3.7 & 80 & 2.1 & 228 & 2.8 \\
\hline Oriental & 22 & 0.6 & 13 & 0.3 & 35 & 0.4 \\
\hline Native Brazilian & 9 & 0.2 & 7 & 0.2 & 16 & 0.2 \\
\hline Total & 4,041 & 51.1 & 3,874 & 48.9 & 7,915 & 100.0 \\
\hline
\end{tabular}


Table 2

Prevalence of the incomplete follow-up on child care according to demographic, socioeconomic, maternal and children's variables, in different periods, in the Northeast Region. Brazil, 2010.

\begin{tabular}{|c|c|c|c|c|c|c|c|}
\hline \multirow[t]{4}{*}{ Variables } & \multicolumn{7}{|c|}{ Northeast Region } \\
\hline & 15 days & 1 month & 2 months & 4 months & 6 months & 9 months & 12 months \\
\hline & $p$-value & $p$-value & $p$-value & $p$-value & $p$-value & $p$-value & $p$-value \\
\hline & $\%(\mathrm{Cl} 95 \%)$ & $\%(\mathrm{Cl} 95 \%)$ & $\%(\mathrm{Cl} 95 \%)$ & $\%(\mathrm{Cl} 95 \%)$ & $\%(\mathrm{Cl} 95 \%)$ & $\%(\mathrm{Cl} 95 \%)$ & $\%(\mathrm{Cl} 95 \%)$ \\
\hline \multicolumn{8}{|c|}{$\begin{array}{l}\text { Municipal size in thou- } \\
\text { sands inhabitants }\end{array}$} \\
\hline$(n=4,041) *$ & 0.683 & 0.020 & 0.095 & 0.286 & 0.289 & 0.759 & 0.166 \\
\hline 10 to 29 & $24.1(22.8-25.5)$ & $34.6(33.1-36.1)$ & $32.4(30.9-33.9)$ & $33.3(31.8-34.8)$ & $31.7(30.3-33.2)$ & $35.9(34.4-37.4)$ & $37.1(35.5-38.6)$ \\
\hline 30 to 49 & $17.9(16.7-19.1)$ & $18.7(17.5-19.9)$ & $18.6(17.4-19.9)$ & $18.4(17.1-19.6)$ & $20.1(18.8-21.4)$ & $20.6(19.3-21.9)$ & $24.1(22.7-25.5)$ \\
\hline 50 to 99 & $14.8(13.7-15.9)$ & $52.6(51.0-54.2)$ & $56.5(54.9-58.1)$ & $56.1(54.5-57.6)$ & $54.3(52.7-55.8)$ & $54.7(53.1-56.3)$ & $50.8(49.2-52.4)$ \\
\hline 100 to 999 & $20.9(19.6-22.2)$ & $22.7(21.4-24.1)$ & $22.9(21.6-24.2)$ & $24.1(22.7-25.5)$ & $24.3(22.9-25.7)$ & $27.5(26.1-28.9)$ & $27.9(26.5-29.3)$ \\
\hline \multicolumn{8}{|c|}{ Economic classification } \\
\hline$A$ and $B$ & $16.4(15.2-17.6)$ & $16.7(15.5-17.9)$ & $13.4(12.3-14.5)$ & $13.6(12.5-14.7)$ & $15.5(14.4-16.8)$ & $16.3(15.1-17.5)$ & $19.3(17.9-20.6)$ \\
\hline $\mathrm{C}$ & $19.4(18.1-20.7)$ & $22.6(21.2-23.9)$ & $22.8(21.4-24.2)$ & $22.8(21.4-24.2)$ & $23.1(21.7-24.5)$ & $25.4(23.9-26.8)$ & $26.6(25.2-28.1)$ \\
\hline $\mathrm{D}$ and $\mathrm{E}$ & $22.1(20.7-23.5)$ & $29.3(27.8-30.8)$ & $29.8(28.3-31.3)$ & $31.4(29.8-32.9)$ & $31.4(29.8-32.9)$ & $34.3(32.7-35.8)$ & $34.8(33.2-36.4)$ \\
\hline
\end{tabular}

Per capita income in

minimum wages quar-

tiles $(n=3,601)$ *

$<0.237$

0.238 to 0.431

0.432 to 0.823

$>0.824$

0.001

$<0.000$

$<0.000$

$<0.000$

0.002

$<0.000$

$\begin{array}{lllllll}22.2(20.8-23.6) & 27.0(25.5-28.5) & 27.2(25.7-28.7) & 27.2(25.7-28.7) & 28.2(26.6-29.7) & 30.0(28.5-31.6) & 31.6(30.0-33.2)\end{array}$

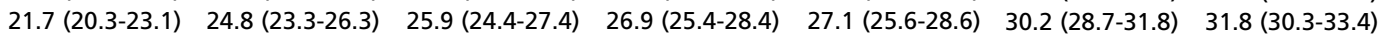

$\begin{array}{llllllll}17.5(16.3-18.8) & 23.2(21.8-24.6) & 22.2(21.4-24.2) & 23.4(22.0-24.8) & 24.6(23.2-26.1) & 26.2(24.7-27.7) & 26.5(25.0-28.0)\end{array}$

$16.1(14.9-17.4) \quad 19.1(17.8-20.4) \quad 18.0(16.7-19.3) \quad 17.8(16.5-19.1) \quad 17.9(16.6-19.2) \quad 22.6(21.2-24.0) \quad 23.1(21.7-24.5)$

Family Welfare

Program (Bolsa Família) $(n=3,973)$ **

Yes

No

0.139

0.097

0.259

0.441

0.310

$20.3(19.0-21.6) \quad 25.0(23.6-26.4) \quad 26.0(24.6-27.4) \quad 26.9(25.4-28.2) \quad 26.9(25.4-28.2) \quad 29.2(27.7-30.7) \quad 30.3(28.8-31.8)$ $20.2(18.9-21.5) \quad 24.7(23.3-26.1) \quad 23.9(22.6-25.3) \quad 24.5(23.1-25.9) \quad 25.3(23.9-26.7) \quad 28.0(26.6-29.4) \quad 28.7(27.3-30.2)$

Maternal age in years $(n=2,989)$ *

19 or less

20 to 29

30 to 39

40 or more

0.922

0.065

0.140

0.179

0.103

0.396

0.084

$21.0(19.5-22.5) \quad 20.9(19.4-22.5) \quad 21.0(19.5-22.5) \quad 23.8(22.2-25.4) \quad 19.4(18.0-20.9) \quad 24.2(22.7-25.8) \quad 22.0(20.5-23.6)$

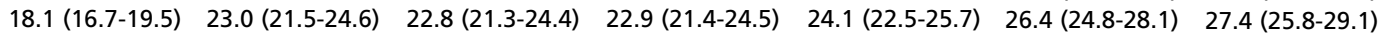

$\begin{array}{llllllll}17.6(16.2-19.0) & 23.6(22.1-25.2) & 23.5(22.0-25.1) & 23.8(22.2-25.4) & 23.7(22.1-25.3) & 26.4(24.8-28.1) & 27.4(25.8-29.1)\end{array}$

$22.4(20.9-24.0) \quad 30.3(28.6-32.0) \quad 28.0(26.4-29.7) \quad 30.2(28.5-31.9) \quad 28.9(27.2-30.6) \quad 28.9(27.2-30.6) \quad 31.5(29.8-33.3)$

Maternal skin color

$(\mathrm{n}=2,988) * *$

White

Mixed

Black

Oriental

Native Brazilian

0.714

0.700

0.749

0.396

18.9 (17.5-20.4) $24.6(23.0-26.2) \quad 23.9(22.3-25.5) \quad 24.3(22.7-25.9) \quad 24.2(22.6-25.8) \quad 28.2(26.6-29.9) \quad 27.2(25.6-28.9)$

$18.7(17.3-20.1) \quad 23.2(21.7-24.8) \quad 23.0(21.5-24.6) \quad 23.9(22.3-25.5) \quad 23.6(22.1-25.3) \quad 25.8(24.2-27.5) \quad 27.2(25.6-28.9)$

$15.8(14.5-17.2) \quad 22.4(20.9-24.0) \quad 22.3(20.8-23.9) \quad 19.7(18.3-21.2) \quad 23.7(22.1-25.3) \quad 25.4(23.8-27.0) \quad 27.2(25.6-28.9)$

$\begin{array}{llllllll}15.4(14.1-16.7) & 25.0(23.4-26.6) & 25.0(23.4-26.6) & 33.3(31.6-35.1) & 50.0(48.2-51.8) & 41.7(39.9-43.5) & 33.3(31.6-35.1)\end{array}$

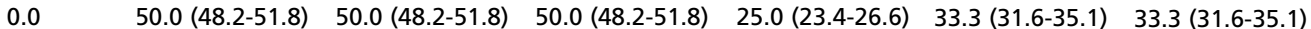

Maternal schooling in years $(n=2,792)$ *

4 or less

5 to 8

9 or more

Presence of partner $(n=2,984)$ **

Yes

No $\begin{array}{llllll}0.049 & 0.002 & <0.000 & <0.000 & 0.002 & <0.000\end{array}$ $20.0(18.5-21.6) \quad 26.6(24.9-28.3) \quad 29.0(27.3-30.7) \quad 28.5(26.8-30.2) \quad 25.9(24.2-27.6) \quad 31.7(29.9-33.5) \quad 34.0(32.2-35.8)$ $20.3(18.8-21.9) \quad 25.5(23.9-27.2) \quad 23.6(22.0-25.2) \quad 25.8(24.2-27.5) \quad 26.8(25.1-28.5) \quad 28.6(26.9-30.4) \quad 30.2(28.5-32.0)$ $16.8(15.4-18.3) \quad 20.6(19.1-22.2) \quad 20.8(19.3-22.4) \quad 20.1(18.6-21.7) \quad 20.8(19.3-22.4) \quad 22.8(21.2-24.5) \quad 23.1(21.5-24.7)$

$\begin{array}{llllllll}0.098 & 0.318 & 0.254 & 0.093 & 0.232 & 0.279 & 0.233\end{array}$

$17.7(16.3-19.1) \quad 22.9(21.4-24.5) \quad 22.5(21.0-24.1) \quad 22.8(21.3-24.4) \quad 23.3(21.8-24.9) \quad 25.7(24.1-27.3) \quad 26.5(24.9-28.2)$ $20.5(19.0-22.0) \quad 24.7(23.1-26.3) \quad 24.6(23.0-26.2) \quad 25.9(24.3-27.6) \quad 25.5(23.9-27.1) \quad 27.8(26.2-29.5) \quad 28.8(27.1-30.5)$ 
Table 2

conclusion

Prevalence of the incomplete follow-up on child care according to demographic, socioeconomic, maternal and children's variables, in different periods, in the Northeast Region. Brazil, 2010.

\begin{tabular}{|c|c|c|c|c|c|c|c|}
\hline \multirow[t]{4}{*}{ Variables } & \multicolumn{7}{|c|}{ Northeast Region } \\
\hline & 15 days & 1 month & 2 months & 4 months & 6 months & 9 months & 12 months \\
\hline & $p$-value & $p$-value & $p$-value & $p$-value & $p$-value & $p$-value & $p$-value \\
\hline & $\%(\mathrm{Cl} 95 \%)$ & $\%(\mathrm{C} 195 \%)$ & $\%(\mathrm{Cl} 95 \%)$ & $\%(\mathrm{Cl} 95 \%)$ & $\%(\mathrm{Cl} 95 \%)$ & $\%(\mathrm{Cl} 95 \%)$ & $\%(\mathrm{Cl} 95 \%)$ \\
\hline
\end{tabular}

Number of prenatal consultations $(n=3,587)$ *

Up to 5

6 or more

Number of live births $(n=2,994)$ *

1

3 or more

Child's sex $(n=4,041)^{\text {** }}$

Male

Female

Child's age in years

$(n=4,041)$ *

1

2

3

4

Child's skin color $(n=3,994)$ **

White

Mixed

Black

Oriental

Native Brazilian

Total
$<0.000$
$<0.000$
$<0.000$
$<0.000$
$<0.000$
$<0.000$
$<0.000$

$24.7(23.3-26.2) \quad 30.4(28.9-31.9) \quad 32.4(30.8-34.0) \quad 32.8(31.2-34.4) \quad 33.0(31.4-34.6) \quad 36.6(35.0-38.2) \quad 38.4(36.8-40.0)$ $18.0(16.7-19.3) \quad 22.7(21.3-24.1) \quad 22.2(20.8-23.6) \quad 23.2(21.8-24.6) \quad 23.6(22.2-25.0) \quad 25.8(24.4-27.3) \quad 26.6(25.1-28.1)$

\subsection{6}

0.037

$<0.000$

0.003

0.001

$<0.000$

$18.7(17.3-20.2) \quad 22.8(21.3-24.4) \quad 22.0(20.5-23.6) \quad 21.3(19.8-22.8) \quad 22.2(20.7-23.8) \quad 24.3(22.7-25.9) \quad 24.7(23.1-26.3)$

$15.2(13.9-16.6) \quad 22.2(20.7-23.8) \quad 22.1(20.6-23.7) \quad 23.0(21.5-24.6) \quad 22.7(21.2-24.3) \quad 25.5(23.9-27.1) \quad 26.5(24.9-28.2)$

$22.3(20.8-23.8) \quad 26.5(24.9-28.2) \quad 26.7(25.1-28.4) \quad 29.4(27.7-31.1) \quad 28.7(27.1-30.4) \quad 31.7(30.0-33.5) \quad 33.1(31.4-34.8)$

$\begin{array}{lllllll}0.720 & 0.075 & 0.158 & 0.016 & 0.372 & 0.210 & 0.236\end{array}$ $19.9(18.6-21.2) \quad 23.6(22.3-25.0) \quad 24.0(22.7-25.4) \quad 24.1(22.8-25.5) \quad 25.3(23.9-26.7) \quad 27.6(26.2-29.0) \quad 28.5(27.1-30.0)$ $20.4(19.1-21.7) \quad 26.1(24.7-27.5) \quad 26.0(24.6-27.4) \quad 27.4(26.0-28.8) \quad 26.6(25.2-28.0) \quad 29.4(28.0-30.9) \quad 30.3(28.8-31.8)$

$\begin{array}{ccccccc}0.071 & 0.201 & 0.662 & 0.696 & 0.809 & 0.612 & 0.112 \\ 22.0(20.7-23.3) & 25.6(24.2-27.0) & 26.3(24.9-27.7) & 25.8(24.4-27.2) & 26.1(24.7-27.5) & 28.6(27.2-30.1) & 31.3(29.8-32.8) \\ 20.1(18.9-21.4) & 24.9(23.6-26.3) & 23.4(22.1-24.8) & 25.8(24.4-27.2) & 25.5(24.1-26.9) & 28.2(26.8-29.7) & 29.1(27.7-30.6) \\ 19.8(18.6-21.1) & 26.2(24.8-27.6) & 25.9(24.5-27.3) & 26.6(25.2-28.0) & 27.3(25.9-28.7) & 30.5(29.0-32.0) & 29.2(27.7-30.7) \\ 18.6(17.4-19.9) & 22.4(21.1-23.7) & 24.5(23.1-25.9) & 24.6(23.2-26.0) & 24.9(23.5-26.3) & 26.6(25.2-28.0) & 27.8(26.4-29.3)\end{array}$

0.120

0.427

0.020

0.075

0.062

0.214

$20.4(19.1-21.7) \quad 25.9(24.5-27.3) \quad 25.0(23.6-26.4) \quad 25.5(24.1-26.9) \quad 26.8(25.4-28.2) \quad 28.5(27.1-30.0) \quad 28.5(27.1-30.0)$

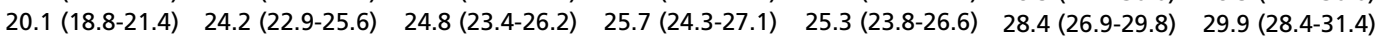

$17.6(16.4-18.8) \quad 21.8(20.5-23.2) \quad 25.9(24.5-27.3) \quad 23.2(21.9-24.6) \quad 27.5(26.0-28.9) \quad 26.1(24.6-27.5) \quad 24.6(23.3-26.0)$

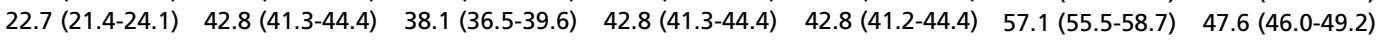

$44.4(42.8-45.9) \quad 44.4(42.8-45.9) \quad 44.4(42.8-45.9) \quad 66.6(65.1-68.1) \quad 55.5(54.0-57.1) \quad 33.3(31.8-34.8) \quad 33.3(31.8-34.8)$

$20.1(19.2-21.0) \quad 24.8(23.8-25.8) \quad 25.0(24.0-26.0) \quad 25.7(24.7-26.7) \quad 25.9(25.0-26.9) \quad 28.5(27.5-29.5) \quad 29.4(28.4-30.5)$

* Chi-square test for linear tendency; ** Chi-square test for heterogeneity. 
Table 3

Prevalence of the incomplete follow-up on child care according to demographic, socioeconomic, maternal and children's variables, in different periods, in the South Region. Brazil, 2010

\begin{tabular}{|c|c|c|c|c|c|c|c|}
\hline \multirow[t]{4}{*}{ Variables } & \multicolumn{7}{|c|}{ South Region } \\
\hline & 15 days & 1 month & 2 months & 4 months & 6 months & 9 months & 12 months \\
\hline & $p$-value & $p$-value & $p$-value & $p$-value & $p$-value & $p$-value & $p$-value \\
\hline & $\%(\mathrm{Cl} 95 \%)$ & $\%(\mathrm{Cl} 95 \%)$ & $\%(\mathrm{Cl} 95 \%)$ & $\%(\mathrm{Cl} 95 \%)$ & $\%(\mathrm{Cl} 95 \%)$ & $\%(\mathrm{Cl} 95 \%)$ & $\%(\mathrm{Cl} 95 \%)$ \\
\hline \multicolumn{8}{|l|}{$\begin{array}{l}\text { Municipal size in thou- } \\
\text { sands inhabitants }\end{array}$} \\
\hline$(n=3,874) *$ & $<0.000$ & $<0.000$ & $<0.000$ & $<0.000$ & $<0.000$ & $<0.000$ & $<0.000$ \\
\hline 10 to 29 & $8.6(7.7-9.5)$ & $13.4(12.3-14.6)$ & $8.4(7.5-9.3)$ & $10.6(9.6-11.6)$ & $6.2(5.4-7.0)$ & $11.7(10.6-12.7)$ & $12.2(11.2-13.3)$ \\
\hline 30 to 49 & $10.5(9.5-11.5)$ & $14.4(13.3-15.6)$ & $16.6(15.4-17.8)$ & $17.4(16.2-18.6)$ & $15.2(14.1-16.4)$ & $20.0(18.7-21.3)$ & $18.6(17.3-19.9)$ \\
\hline 50 to 99 & $4.1(3.5-4.8)$ & $8.1(7.2-9.0)$ & $9.4(8.5-10.4)$ & $9.9(8.9-10.9)$ & $9.3(8.4-10.3)$ & $12.5(11.4-13.6)$ & $12.2(11.2-13.3)$ \\
\hline 100 to 999 & $5.4(4.7-6.2)$ & $4.1(3.5-4.8)$ & $4.9(4.2-5.6)$ & $4.7(4.0-5.4)$ & $5.3(4.6-6.1)$ & $7.9(7.0-8.8)$ & $7.8(6.9-8.7)$ \\
\hline \multicolumn{8}{|l|}{ Economic classification } \\
\hline$(n=3,681) *$ & $<0.000$ & $<0.000$ & $<0.000$ & $<0.000$ & $<0.000$ & $<0.000$ & $<0.000$ \\
\hline$A$ and $B$ & $3.6(30.4-42.9)$ & $4.8(4.1-5.6)$ & $6.0(5.2-6.8)$ & $5.0(4.3-5.7)$ & $5.5(4.7-6.3)$ & $8.2(7.3-9.1)$ & $7.7(6.8-8.6)$ \\
\hline $\mathrm{C}$ & $6.8(6.0-7.7)$ & $7.6(6.7-8.5)$ & $8.0(7.1-8.9)$ & $9.3(8.3-10.3)$ & $8.3(7.4-9.3)$ & $11.5(10.4-12.6)$ & $11.8(10.7-12.9)$ \\
\hline $\mathrm{D}$ and $\mathrm{E}$ & $12.6(11.5-13.7)$ & $14.9(13.7-16.1)$ & $15.7(14.5-16.9)$ & $15.8(14.6-17.1)$ & $13.2(12.1-14.4)$ & $20.2(18.9-21.7)$ & $17.1(15.8-18.4)$ \\
\hline \multicolumn{8}{|l|}{ Per capita income in } \\
\hline $\begin{array}{l}\text { minimum wages quar- } \\
\text { tiles }(n=3,485)^{\star}\end{array}$ & $<0.000$ & $<0.000$ & $<0.000$ & $<0.000$ & $<0.000$ & $<0.000$ & $<0.000$ \\
\hline$<0.237$ & $11.7(10.6-12.8)$ & $15.7(14.5-16.9)$ & $16.5(15.2-17.8)$ & $18.8(17.5-20.2)$ & $16.9(15.6-18.2)$ & $21.3(19.9-22.7)$ & $20.1(18.7-21.5)$ \\
\hline 0.238 to 0.431 & $8.5(7.7-9.6)$ & $9.8(8.8-10.9)$ & $10.4(9.4-11.5)$ & $11.1(10.0-12.2)$ & $9.2(8.2-10.2)$ & $15.6(14.4-16.9)$ & $13.9(12.7-15.1)$ \\
\hline 0.432 to 0.823 & $6.2(5.4-7.1)$ & $7.1(6.2-8.0)$ & $7.8(6.9-8.7)$ & $8.4(7.5-9.4)$ & $8.1(7.2-9.1)$ & $10.3(9.3-11.4)$ & $10.7(9.6-11.8)$ \\
\hline$>0.824$ & $4.4(3.7-5.1)$ & $4.5(3.8-5.2)$ & $5.8(5.0-6.6)$ & $5.5(4.7-6.3)$ & $5.2(4.5-6.0)$ & $7.8(6.9-8.8)$ & $7.8(6.9-8.8)$ \\
\hline \multicolumn{8}{|l|}{ Family Welfare } \\
\hline \multicolumn{8}{|l|}{ Program (Bolsa Família) } \\
\hline$(n=3,804) * *$ & $<0.000$ & $<0.000$ & $<0.000$ & $<0.000$ & 0.001 & $<0.000$ & 0.001 \\
\hline Yes & $10.8(9.8-11.8)$ & $10.8(9.8-11.8)$ & $11.9(10.8-13.0)$ & $12.9(11.8-14.1)$ & $11.1(10.1-12.2)$ & $15.5(14.3-16.7)$ & $14.5(13.4-15.7)$ \\
\hline No & $5.3(4.6-6.7)$ & $6.6(5.8-7.4)$ & $7.3(6.5-8.2)$ & $7.4(6.6-8.3)$ & $7.2(6.4-8.1)$ & $10.3(9.3-11.3)$ & $10.2(9.2-11.2)$ \\
\hline \multicolumn{8}{|l|}{ Maternal age in years } \\
\hline 19 or less & $10.0(8.9-11.2)$ & $9.7(8.6-10.8)$ & $11.2(10.1-12.5)$ & $10.7(9.6-12.0)$ & $9.7(8.6-10.9)$ & $13.3(12.0-14.6)$ & $11.4(10.2-12.6)$ \\
\hline 20 to 29 & $5.3(4.5-6.2)$ & $7.3(6.4-8.3)$ & $7.7(6.7-8.7)$ & $7.7(6.7-8.7)$ & $7.1(6.2-8.1)$ & $10.2(9.1-11.4)$ & $10.7(9.6-11.9)$ \\
\hline 30 to 39 & $5.3(4.5-6.2)$ & $4.6(3.8-5.4)$ & $5.2(4.4-6.1)$ & $5.5(4.7-6.4)$ & $5.5(4.7-6.4)$ & $7.8(6.8-8.9)$ & $7.7(6.7-8.7)$ \\
\hline 40 or more & $6.1(5.2-7.1)$ & $9.0(8.0-10.1)$ & $9.7(8.6-10.9)$ & $9.0(8.0-10.1)$ & $8.2(7.2-9.3)$ & $11.7(10.5-13.0)$ & $10.4(9.3-11.6)$ \\
\hline \multicolumn{8}{|l|}{ Maternal skin color } \\
\hline$(n=2,873) * *$ & 0.141 & $<0.000$ & 0.333 & 0.005 & 0.059 & 0.060 & 0.125 \\
\hline White & $5.8(5.0-6.7)$ & $6.1(5.2-7.1)$ & $7.0(6.1-8.0)$ & $6.5(5.6-7.5)$ & $6.2(5.3-7.2)$ & $9.1(8.0-10.2)$ & $9.2(8.1-10.3)$ \\
\hline Mixed & $5.1(4.9-5.3)$ & $7.9(7.7-8.0)$ & $7.9(7.7-8.0)$ & $9.1(8.9-9.2)$ & $7.9(7.7-8.0)$ & $11.4(10.2-12.6)$ & $10.7(9.5-11.9)$ \\
\hline Black & $7.1(6.9-7.3)$ & $4.9(4.7-5.1)$ & $6.2(6.0-6.4)$ & $7.5(7.3-7.6)$ & $11.3(10.1-12.5)$ & $8.7(8.6-8.9)$ & $8.6(8.5-8.8)$ \\
\hline Oriental & 0.0 & 0.0 & 0.0 & 0.0 & 0.0 & $8.3(8.2-8.5)$ & $8.3(8.2-8.5)$ \\
\hline Native Brazilian & $25.0(23.4-26.6)$ & $44.4(42.5-46.3)$ & $22.2(20.6-23.8)$ & $33.3(31.5-35.1)$ & $22.2(20.7-23.8)$ & $33.3(31.5-35.1)$ & $33.3(31.5-35.1)$ \\
\hline \multicolumn{8}{|l|}{ Maternal schooling in } \\
\hline years $(n=2,790)$ * & $<0.000$ & $<0.000$ & $<0.000$ & $<0.000$ & $<0.000$ & $<0.000$ & 0.010 \\
\hline 4 or less & $9.6(8.5-10.8)$ & $7.9(6.9-9.0)$ & $11.1(9.9-12.4)$ & $11.0(9.8-12.3)$ & $8.3(7.3-9.4)$ & $14.9(13.6-16.3)$ & $13.7(12.4-15.1)$ \\
\hline 5 to 8 & $6.8(5.9-7.8)$ & $8.7(7.7-9.8)$ & $8.7(7.6-9.8)$ & $8.7(7.7-9.9)$ & $8.8(7.7-9.3)$ & $12.0(10.8-13.3)$ & $9.9(8.8-11.1)$ \\
\hline 9 or more & $4.3(3.6-5.1)$ & $4.8(4.0-5.7)$ & $5.3(4.5-6.2)$ & $5.3(4.5-6.2)$ & $4.9(4.1-5.8)$ & $7.1(6.2-8.2)$ & $8.5(7.5-9.6)$ \\
\hline \multicolumn{8}{|l|}{ Presence of partner } \\
\hline$(n=2,896) * *$ & 0.376 & 0.251 & 0.123 & 0.562 & 0.654 & 0.524 & 0.962 \\
\hline Yes & $5.5(4.7-6.4)$ & $6.4(5.5-7.4)$ & $6.9(6.0-7.9)$ & $7.1(6.2-8.1)$ & $6.7(5.8-7.7)$ & $9.5(8.4-10.7)$ & $9.6(8.5-10.6)$ \\
\hline No & $6.6(5.7-7.6)$ & $7.8(6.8-8.9)$ & $8.9(7.9-10.0)$ & $7.9(6.9-9.0)$ & $7.3(6.4-8.4)$ & $10.5(9.4-11.7)$ & $9.6(8.5-10.6)$ \\
\hline
\end{tabular}

\footnotetext{
* Chi-square test for linear tendency; ** Chi-square test for heterogeneity.
} 
Table 3

conclusion

Prevalence of the incomplete follow-up on child care according to demographic, socioeconomic, maternal and children's variables, in different periods, in the South Region. Brazil, 2010.

\begin{tabular}{|c|c|c|c|c|c|c|c|}
\hline \multirow[t]{4}{*}{ Variables } & \multicolumn{7}{|c|}{ South Region } \\
\hline & 15 days & 1 month & 2 months & 4 months & 6 months & 9 months & 12 months \\
\hline & $p$-value & $p$-value & $p$-value & $p$-value & $p$-value & $p$-value & $p$-value \\
\hline & $\%(\mathrm{Cl} 95 \%)$ & $\%(\mathrm{Cl} 95 \%)$ & $\%(\mathrm{Cl} 95 \%)$ & $\%(\mathrm{Cl} 95 \%)$ & $\%(\mathrm{Cl} 95 \%)$ & $\%(\mathrm{Cl} 95 \%)$ & $\%(\mathrm{Cl} 95 \%)$ \\
\hline \multicolumn{8}{|l|}{$\begin{array}{l}\text { Number of prenatal } \\
\text { consultations }\end{array}$} \\
\hline$(n=3,515)^{*}$ & $<0.000$ & $<0.000$ & $<0.000$ & $<0.000$ & $<0.000$ & $<0.000$ & $<0.000$ \\
\hline Up to 5 & $11.8(10.7-12.9)$ & $14.4(13.3-15.6)$ & $16.4(15.2-17.7)$ & $16.2(15.0-17.5)$ & $14.1(13.0-15.3)$ & $20.8(19.4-22.2)$ & $18.7(17.4-20.1)$ \\
\hline 6 or more & $5.9(5.1-6.7)$ & $6.7(5.9-7.6)$ & $7.1(6.3-8.0)$ & $7.4(6.6-8.4)$ & $7.1(6.3-8.0)$ & $10.1(9.1-11.2)$ & $10.1(9.1-11.2)$ \\
\hline \multicolumn{8}{|l|}{ Number of live births } \\
\hline$(n=2,899)^{*}$ & 0.028 & 0.013 & $<0.000$ & 0.001 & $<0.000$ & $<0.000$ & 0.008 \\
\hline 1 & $5.1(4.3-6.0)$ & $6.1(5.2-7.1)$ & $6.1(5.2-7.1)$ & $6.2(5.3-7.2)$ & $5.7(4.9-6.6)$ & $8.0(7.0-9.1)$ & $9.0(8.0-10.1)$ \\
\hline 2 & $5.0(4.2-5.9)$ & $5.4(4.6-6.3)$ & $5.7(4.9-6.6)$ & $6.3(5.4-7.3)$ & $5.9(5.0-6.8)$ & $8.5(7.5-9.6)$ & $8.0(7.0-9.1)$ \\
\hline 3 or more & $7.7(6.7-8.7)$ & $9.5(8.4-10.6)$ & $11.5(10.3-12.8)$ & $10.7(9.6-11.9)$ & $10.3(9.2-11.5)$ & $14.7(13.4-16.1)$ & $13.4(12.2-14.7)$ \\
\hline Child's sex $(n=3,874)^{* *}$ & 0.381 & 0.775 & 0.367 & 0.900 & 0.847 & 0.794 & 0.144 \\
\hline Male & $6.7(5.9-7.5)$ & $7.4(6.6-8.3)$ & $8.6(7.7-9.5)$ & $8.6(7.7-9.5)$ & $8.0(7.1-8.9)$ & $11.2(10.2-12.3)$ & $11.7(10.7-12.8)$ \\
\hline Female & $6.0(5.3-6.8)$ & $7.7(6.9-8.6)$ & $7.8(7.0-8.7)$ & $8,4(7.5-9.3)$ & $7.9(7.0-8.8)$ & $11.5(10.5-12.6)$ & $10.2(9.2-11.2)$ \\
\hline \multicolumn{8}{|l|}{ Child's age in years } \\
\hline$(n=3,874)^{*}$ & 0.830 & 0.227 & 0.318 & 0.430 & 0.950 & 0.890 & 0.029 \\
\hline 1 & $6.6(5.8-7.4)$ & $6.0(5.3-6.8)$ & $7.3(6.5-8.2)$ & $8.1(7.2-9.0)$ & $7.3(6.5-8.2)$ & $10.6(9.6-11.6)$ & $12.9(11.8-14.0)$ \\
\hline 2 & $6.0(5.3-6.8)$ & $9.1(8.2-10.1)$ & $9.0(8.1-10.0)$ & $9.9(8.9-10.9)$ & $9.3(8.4-10.3)$ & $13.0(11.9-14.1)$ & $10.8(9.8-11.8)$ \\
\hline 3 & $6.8(6.0-7.7)$ & $7.4(6.6-8.3)$ & $7.7(6.8-8.6)$ & $8.8(7.9-9.8)$ & $7.6(6.7-8.5)$ & $11.5(10.5-12.6)$ & $10.2(9.2-11.2)$ \\
\hline 4 & $6.1(5.3-6.9)$ & $8.0(7.1-8.9)$ & $9.0(8.1-10.0)$ & $7.3(6.5-8.2)$ & $7.7(6.8-8.6)$ & $10.7(9.7-11.8)$ & $9.9(8.9-10.9)$ \\
\hline \multicolumn{8}{|l|}{ Child's skin color } \\
\hline$(n=3,994) * *$ & 0.120 & 0.198 & 0.037 & 0.103 & 0.072 & 0.164 & 0.235 \\
\hline White & $6.5(5.7-7.3)$ & $7.4(6.6-8.3)$ & $7.7(6.8-8.6)$ & $8.1(7.2-9.0)$ & $7.7(6.8-8.6)$ & $11.0(10.0-12.1)$ & $10.6(9.6-11.6)$ \\
\hline Mixed & $5.7(5.5-5.8)$ & $8.1(7.9-8.2)$ & $10.1(9.1-11.1)$ & $9.9(9.8-9.9)$ & $9.1(8.9-9.1)$ & $12.1(11.1-13.2)$ & $12.0(10.9-13.1)$ \\
\hline Black & $6.5(6.3-6.6)$ & $6.8(6.6-6.9)$ & $6.8(6.6-6.9)$ & $6.7(6.6-6.9)$ & $5.4(5.2-5.5)$ & $14.8(13.7-16.0)$ & $12.1(11.1-13.2)$ \\
\hline Oriental & 0.0 & 0.0 & $7.6(7.5-7.8)$ & $7.7(7.5-7.8)$ & 0.0 & $23.1(21.7-24.4)$ & $23.1(21.7-24.4)$ \\
\hline Native Brazilian & $28.5(27.1-30.0)$ & $28.5(27.1-30.0)$ & 33.3 (31.8-34.8) & 33.3 (31.8-34.8) & $33.3(31.8-34.8)$ & 33.3 (31.8-34.8) & $28.5(27.1-30.0)$ \\
\hline Total & $6.4(5.9-7.0)$ & $7.5(6.9-8.1)$ & $8.2(7.6-8.8)$ & $8.5(7.9-9.2)$ & $8.0(7.4-8.6)$ & $11.4(10.7-12.1)$ & $11.0(10.3-11.7)$ \\
\hline
\end{tabular}

* Chi-square test for linear tendency; ** Chi-square test for heterogeneity. 
Table 4

Prevalence, crude and adjusted analysis of the factors associated to the incomplete follow-up on child care in the Northeast and in the South Regions, Brazil, 2010.

\begin{tabular}{|c|c|c|c|c|c|c|}
\hline \multirow[t]{4}{*}{ Variables } & \multicolumn{3}{|c|}{ Northeast } & \multicolumn{3}{|c|}{ South } \\
\hline & Prevalence & Crude PR* & Adjusted PR* & Prevalence & Crude $\mathrm{PR}^{*}$ & Adjusted PR* \\
\hline & & $p$-value & $p$-value & $p$-value & $p$-value & $p$-value \\
\hline & $\%(\mathrm{Cl} 95 \%)$ & PR (Cl95\%) & PR (Cl95\%) & $\%(\mathrm{Cl} 95 \%)$ & PR (Cl95\%) & PR (Cl95\%) \\
\hline $\begin{array}{l}\text { Municipal size in thou- } \\
\text { sands inhabitants }\end{array}$ & & $<0.000$ & $<0.000$ & & $<0.000$ & $<0.000$ \\
\hline 10 to 29 & $59.2(57.6-60.7)$ & 1.0 & 1.0 & $33.7(32.2-35.2)$ & 1.0 & 1.0 \\
\hline 30 to 49 & $45.0(43.4-46.5)$ & $0.76(0.67-0.84)$ & $0.72(0.64-0.82)$ & $40.1(38.5-41.7)$ & $1.19(0.98-1.43)$ & $1.14(0.94-1.39)$ \\
\hline 50 to 99 & $73.6(72.2-74.9)$ & $1.24(1.10-1.39)$ & $1.22(1.08-1.39)$ & $28.6(27.2-30.1)$ & $0.84(0.69-1.03)$ & $0.80(0.65-1.00)$ \\
\hline 100 to 999 & $53.7(52.1-55.2)$ & $0.90(0.82-0.99)$ & $0.91(0.82-1.01)$ & $23.4(22.1-24.8)$ & $0.69(0.57-0.82)$ & $0.69(0.57-0.84)$ \\
\hline Economic classification & & $<0.000$ & $<0.000$ & & $<0.000$ & $<0.000$ \\
\hline$A$ and $B$ & $38.5(36.9-40.1)$ & 1.0 & 1.0 & $20.2(18.9-21.5)$ & 1.0 & 1.0 \\
\hline $\mathrm{C}$ & $51.0(49.4-52.6)$ & $1.32(1.14-1.53)$ & $1.22(1.04-1.44)$ & $29.9(28.4-31.4)$ & $1.47(1.30-1.68)$ & $1.32(1.14-1.53)$ \\
\hline$D$ and $E$ & $59.2(57.6-60.7)$ & $1.53(1.32-1.78)$ & $1.41(1.19-1.67)$ & $41.5(39.9-43.1)$ & $2.05(1.74-2.41)$ & $1.67(1.37-2.03)$ \\
\hline $\begin{array}{l}\text { Per capita income in } \\
\text { minimum wages quar- }\end{array}$ & & & & & & \\
\hline tiles & & 0.002 & 0.375 & & $<0.000$ & 0.079 \\
\hline$<0.237$ & $55.0(53.4-56.6)$ & 1.0 & 1.0 & $41.1(39.4-42.7)$ & 1.0 & 1.0 \\
\hline 0.238 to 0.431 & $55.8(54.1-57.4)$ & $1.01(0.94-1.08)$ & $1.03(0.95-1.11)$ & $32.6(31.0-34.2)$ & $0.79(0.67-0.93)$ & $0.86(0,72-1.03)$ \\
\hline 0.432 to 0.823 & $51.4(49.7-53.0)$ & $0.93(0.85-1.02)$ & $1.00(0.91-1.10)$ & $27.3(25.8-28.8)$ & $0.66(0.56-0.77)$ & $0.80(0.66-0.96)$ \\
\hline$>0.824$ & $44.9(43.3-46.5)$ & $0.81(0.72-0.92)$ & $0.91(0.80-1.05)$ & $22.4(21.0-23.8)$ & $0.54(0.46-0.63)$ & $0.77(0.63-0.95)$ \\
\hline Family Welfare & & & & & & \\
\hline Program (Bolsa Família) & & 0.703 & & & $<0.000$ & 0.738 \\
\hline Yes & $54.0(52.4-55.5)$ & $1.01(0.95-1.07)$ & & $35.5(34.0-37.0)$ & $1.34(1.20-1.51)$ & $0.97(0.84-1.12)$ \\
\hline No & $53.4(51.8-54.9)$ & 1.0 & & $26.3(24.9-27.7)$ & 1.0 & 1.0 \\
\hline Maternal age in years & & 0.109 & 0.182 & & $<0.000$ & 0.035 \\
\hline 19 or less & $54.8(53.0-56.6)$ & 1.0 & 1.0 & $35.0(33.2-36.8)$ & 1.0 & 1.0 \\
\hline 20 to 29 & $50.1(48.3-51.9)$ & $0.92(0.81-1.03)$ & $0.96(0.84-1.12)$ & $26.2(24.6-27.8)$ & $0.74(0.60-0.91)$ & $0.78(0.61-1.00)$ \\
\hline 30 to 39 & $50.0(48.2-51.8)$ & $0.91(0.80-1.03)$ & $1.02(0.88-1.20)$ & $22.8(21.3-24.4)$ & $0.65(0.52-0.81)$ & $0.72(0.55-0.92)$ \\
\hline 40 or more & $57.8(56.0-59.6)$ & $1.05(0.89-1.25)$ & $1.15(0.93-1.42)$ & $30.2(28.5-31.9)$ & $0.86(0.66-1.11)$ & $0.90(0.67-1.22)$ \\
\hline Maternal skin color & & 0.515 & & & 0.159 & 0.740 \\
\hline White & $51.1(42.3-52.9)$ & 1.0 & & $25.9(24.3-27.5)$ & 1.0 & 1.0 \\
\hline Mixed & $51.4(49.6-53.2)$ & $1.01(0.92-1.09)$ & & $26.3(24.6-27.9)$ & $1.01(0.87-1.16)$ & $0.89(0.76-1.05)$ \\
\hline Black & $48.1(46.3-49.9)$ & $0.94(0.80-1.10)$ & & $27.9(26.2-29.5)$ & $1.07(0.76-1.52)$ & $1.03(0.67-1.57)$ \\
\hline Oriental & $61.5(59.7-63.2)$ & $1.20(0.77-1.86)$ & & $25.0(23.4-26.6)$ & $0.96(0.36-2.57)$ & $1.09(0.43-2.78)$ \\
\hline Native Brazilian & $75.0(73.4-76.5)$ & $1.46(0.82-2.59)$ & & $55.5(53.7-57.3)$ & $2.14(1.19-3.86)$ & $1.20(0.42-3.40)$ \\
\hline $\begin{array}{l}\text { Maternal schooling in } \\
\text { years }\end{array}$ & & 0.003 & 0.086 & & $<0.000$ & 0.085 \\
\hline 4 or less & $52.9(51.0-54.7)$ & $1.14(1.05-1.23)$ & $1.04(0.91-1.19)$ & $32.2(30.5-34.0)$ & $1.47(1.20-1.79)$ & $1.12(0.94-1.32)$ \\
\hline 5 to 8 & $54.3(52.4-56.1)$ & $1.11(0.99-1.24)$ & $1.11(1.02-1.22)$ & $28.6(26.9-30.3)$ & $1.30(1.13-1.49)$ & $1.29(1.02-1.65)$ \\
\hline 9 or more & $47.5(45.6-49.4)$ & 1.0 & 1.0 & $21.9(20.4-23.5)$ & 1.0 & 1.0 \\
\hline Presence of partner & & 0.023 & 0.121 & & 0.007 & 0.051 \\
\hline Yes & 49.7 (47.9-51.5) & $0.91(0.84-0.98)$ & $0.93(0.85-1.01)$ & $24.9(23.3-26.5)$ & $0.81(0.70-0.94)$ & $0.84(0.71-0.99)$ \\
\hline No & $54.4(52.6-56.2)$ & 1.0 & 1.0 & $30.6(28.9-32.3)$ & 1.0 & 1.0 \\
\hline
\end{tabular}

\footnotetext{
* Poisson Regression.
} 
Prevalence, crude and adjusted analysis of the factors associated to the incomplete follow-up on child care in the Northeast and in the South Regions, Brazil, 2010.

\begin{tabular}{|c|c|c|c|c|c|c|}
\hline \multirow[t]{4}{*}{ Variables } & \multicolumn{3}{|c|}{ Northeast } & \multicolumn{3}{|c|}{ South } \\
\hline & Prevalence & Crude $\mathrm{PR}^{*}$ & Adjusted $\mathrm{PR}^{*}$ & Prevalence & Crude PR* & Adjusted PR* \\
\hline & & $p$-value & $p$-value & $p$-value & $p$-value & $p$-value \\
\hline & $\%(\mathrm{Cl} 95 \%)$ & PR (CI95\%) & PR (Cl95\%) & $\%(\mathrm{Cl} 95 \%)$ & PR (CI95\%) & PR (Cl95\%) \\
\hline
\end{tabular}

Number of prenatal consultations Up to 5

6 or more

Number of live births

1

2

3 or more

Child's sex

Male

Female

Child's age in years

1

2

3

4

Child's skin color

White

Mixed

Black

Oriental

Native Brazilian

Total
$62.1(60.5-63.7)$

49.7 (48.1-51.4)

$50.0(48.2-51.8)$

48.8 (47.0-50.6)

$56.1(54.3-57.9)$

53.3 (51.8-54.9)

54.0 (52.4-55.5)

55.9 (54.4-57.4)

$53.0(51.5-54.6)$

$53.9(52.3-55.4)$

$51.6(50.0-53.1)$

52.9 (51.3-54.4)

53.9 (52.4-55.5)

47.9 (46.4-49.5)

$68.1(66.7-69.6)$

$100.0(91.0-109.7)$

$53.6(52.5-54.7)$
$<0.000$

1.0

$0.79(0.74-0.85)$

0.005
1.0

0.97 (0.89-1.06)

$1.12(1.03-1.22)$

0.668

1.0

1.01 (0.95-1.07)

0.260

1.0

$0.94(0.87-1.02)$

$0.96(0.89-1.04)$

0.92 (0.84-1.00)

0.662

1.0

$1.02(0.95-1.08)$

$0.90(0.76-1.08)$

$1.28(0.96-1.72)$

1.89 (1.79-1.99)

$1.90(1.79-2.00)$

$<0.000$
1.0
$0.83(0.75-0.92)$
0.048
1.0
$0.92(0.82-1.02)$
$1.07(0.94-1.21)$

$<0.000$

1.0

$2.9(41.3-44.6)$

(2)

$24.1(22.6-25.7)$

22.8 (21.3-24.4)

33.5 (31.8-35.2)

$29.0(27.6-30.4)$

$27.5(26.1-28.9)$

29.5 (28.1-31.0)

$26.9(25.5-28.3)$

27.7 (26.3-29.1)

$28.8(27.4-30.3)$

27.7 (26.3-29.1)

29.7 (28.3-31.2)

$33.7(32.2-35.2)$

$38.4(36.9-40.0)$

42.8 (41.3-44.4)

283 (27.3-29.3)
$0.57(0.50-0.65)$

\section{$<0.000$}

1.0

$0.94(0.81-1.10)$

$1.38(1.20-1.60)$

0.294

1.0

$0.94(0.85-1.04)$

0.594

1.0

$0.91(0.79-105)$

$0.93(0.81-1.07)$

$0.97(0.85-1.11)$

0.351

1.0

$1.07(0.95-1.21)$

$1.22(0.89-1.66)$

$1.39(0.69-2.77)$

$1.55(0.65-3.65)$

$0.52(0.49-0.55)$ was observed in children living in the Northeast and the protective effect for the ones in the South. For both regions, the most associated factors were related to the size of the municipality, the economic classification, and the use of health services.

The total prevalence for the incomplete followup on child care was evidenced in the Northeast and in the South, and it is a great concern on the continuous care characteristics advocated for this stage in the child's life. This result implies that parents or the responsible guardians of the child have not accessed the health services or have encountered some kind of restriction in their access. In the same regions of this present study, another research reported that only one out of five children had gone to at least nine visits in the first two years of life at the health unit in their coverage area. 15 In Minas Gerais State, $24.2 \%$ of the children attended at the ESF were not regularly controlled with child care. 18 In Maranhão State, the access was the worst dimension assessed among the ESF users, ${ }^{19}$ still in the same State, another study identified that $61.4 \%$ of the children did not have any child care consultation. 10 The authors attribute these results to the difference in territory with great geographic distances, besides Maranhão State has many small municipalities, where lots of the programmed actions are not available to the population. ${ }^{10} \mathrm{Such}$ scenario can transmit a reality to health services access1, a historical reflection on the socioeconomic conditions and the organization of services, which contribute to a complex and multi-factorial phenomenon.

In the Brazilian context, the Northeast and the South Regions are known to present discrepancies in their socioeconomic development and in health service management. The Northeast Region is marked by unfavorable survival conditions, in a 
scenario of slow socioeconomic development, and health policies produced and offered in ways that are inconsistent along with the regional necessities 6 ; it is also characterized by a social predominance of the less beneficiary positions, besides presenting the worst indicator in infant mortality in 1990, 2000 and 2007 , in the country 7 and a larger volume to incentive social programs in transferring income to the population. On the other hand, the South region offers better socioeconomic conditions to its population, ${ }^{7}$ thus, it is one of the most developed regions in the country. 8 Studies indicate that individuals living in the Southeast and in the South Regions have a greater chance of using health services when comparing to other regions. 8,20 Thus, the regional differences existing in Brazil are capable of influencing the context to access services by the population with significant inequalities which was corroborated by the data in this study, showing less follow-ups on child care in the Northeast.

Regarding the population size, this study identified in the South region a tendency to reduce the lack of follow-up as the municipal size increased, evidencing by the greater effect in the municipalities with more than 100 thousand inhabitants when compared to those with less than 30 thousand inhabitants. At municipal level that health policies focuses on primary care and are conducted and managed in a scenario that should consider the social and economic peculiarities. The larger municipalities may have greater availability of health services, easier ways in geographical and economic access, more complete care units and qualified health teams, and besides probably having a better educated population, therefore, they are more aware of the necessity to adopt preventive health actions. Such differences among municipalities may influence the access to the services, which may become barriers for the health users. ${ }^{1}$ The medical literature already indicates evidences that individuals living in areas with a better socioeconomic development have better access and the use of the health services. $8,20,21$

On the other hand, this tendency was not identified in the Northeast region. In this region, the municipalities presented variation in the risk and protection effect in the incomplete follow-up on child care. The protective effect was identified in the municipalities with 30 to 49 thousand inhabitants. This observation may reflect in different contexts of the management in the municipal in health services with diversified organizations in the scope of basic care.1,6 However, the municipalities with more than 100 thousand inhabitants also had the same sense of the protective effect as identified in the South
Region, although the statistical significance was not maintained.

The greatest lack of follow-ups on child care were in classes $\mathrm{D}$ and $\mathrm{E}$ in both regions which reinforces the role of economic determinants in the use of the services, even in a public and universal health system. It is worth mentioning that, through the sample strategy, this study included families attended at the basic health units by the National Health Service (Sistema Único de Saúde) and it would not be expected that the lower income families would represent a possible barrier to use the child care services. ${ }^{1}$ However, such iniquities have also been described in many studies in different places in Brazil, mainly related to the use of health care consultations.21-23

Although the South region presents a better socioeconomic conditions and a lower prevalence of lack of follow-ups on child care, this region pointed out the greatest differences among the economic classes and in the income quartile per capita observing by the higher risk expressed in classes $\mathrm{D}$ and $\mathrm{E}$ and the protective effect in families with higher quartile. These findings reinforce the expressive presence of inequalities among economic classes and indicate failures in the strategy of reducing these differences.

Inequities in the income distribution imply considerably in the social and health determinants, $1,24,25,26$ coming from a social stratification 27 and political inequalities that permeate in the health system and challenges its management. ${ }^{2}$ Hence, it is necessary actions that involve the whole political sphere of governments in order to promote social and economic policies capable of reducing income differences and promoting equity in the health system.2,24

In the level of individual maternal determinants in the South region, the maternal age between 30 and 39 years influenced the children's visit to the child care, on the other hand, there seems to be a risk tendency for failures in the care for mothers with more children. In both regions, the access was significantly higher for children whose mothers had more than six prenatal consultations and could have indicated that mothers with more frequency at the health service before the delivery, maintain them during the children's first year of life. Consequently, the possible understanding in health knowledge acquired by these mothers with the continuity of their prenatal care, 13 they would possibly have a better understanding of the importance of this follow-up in which would act as a positive factor for the regularity in the child care. 11 
Although several researches $1,2,24,25$ point out the influence of demographic characteristics in the access to health services, this study did not show any evidence of such effect in relation to individual child factors. Differences related to sex, age and skin color are factors which slowly no longer impact the access to health services, besides indicating advanced progress in the achievement of equity.

One of the weaknesses in this present study is the lack of profound and complete information on reasons why children did not have the recommended child care services. Factors related to geographical, financial, organizational and information barriers, as well as individual beliefs and values may contribute to the non-fulfillment in care. ${ }^{28,29}$ In this study was found that the verification of the child's weight and height by the community health agents during home visit had reverse implications, since many mothers reported considering this procedure sufficient for the health follow-up of their children, which seems to be a misunderstanding by the health services in educational actions for the community and the need in resuming health information.

It is also worth noting that the interviews were carried out up to four years apart from the assessed events, which may result in possible recall failures. The latency between the data collection and the publication of this study should be highlighted, although there were no important transformation in the health policies during this period that could have

\section{References}

1. Assis MMA, Jesus WLAD. Acesso aos serviços de saúde: abordagens, conceitos, políticas e modelo de análise. Ciên Saúde Colet. 2012; 17 (11): 2865-75.

2. Sanchez RM, Ciconelli RM. Conceitos de acesso à saúde. Rev Panam Salud Publica. 2012; 31 (3): 260-8.

3. Travassos C, Martins M. Uma revisão sobre os conceitos de acesso e utilização de serviços de saúde. Cad Saúde Pública. 2004; 20 (Supl. 2): 190-8.

4. Viacava F, Ugá MAD, Porto S, Laguardia J, da Silva Moreira R. Avaliação de Desempenho de Sistemas de Saúde: um modelo de análise. Ciênc Saúde Colet. 2012; 17 (4): 921-34

5. Andersen RM. Revisiting the behavioral model and access to medical care: does it matter? J Health Social Behav. 1995; 36 (1): 1-10

6. Paim J, Travassos C, Almeida C, Bahia L, Macinko J. The Brazilian health system: history, advances, and challenges. Lancet. 2011; 377 (9779): 1778-97.

7. Victora CG, Aquino EML, Leal MC, Monteiro CA, Barros FC, Szwarcwald CL. Maternal and child health in Brazil progress and challenges. Lancet. 2011; 377 (9780): 1863 76. impacted the changes of these results. Although this study does not allow to infer causality because of its cross-sectional design, it is able to determine the direction and magnitude of the associations found. Thus, the findings show the persistence of the inequalities in a universal access to child health services which brings contributions to the access debate and recommendations for the public health management.

Child care is available in most of the UBS, its activities involve follow-up on growth and development, vaccination status, food / nutrition and measurements on early and timely interventions on disease prevention, age-specific diseases and health recovery. These are performed by nurses and physicians, 13 present in most teams, that is, the offer exists, but the use is not fully implemented. However, there is a lack of information about the quality of the services offered, either in terms of physical structure, in health unit organization and professional-health user relationship.

In general, it can be concluded that the regions and the municipalities indicate differences in the follow-up on child care in health services. The inequalities in income distribution continue to reflect differences in the use of the services for the less beneficiary groups. This study also showed that continuity in care seems to contribute in the access to child health in primary care.

8. Travassos C, Oliveira EXD, Viacava F. Desigualdades geográficas e sociais no acesso aos serviços de saúde no Brasil: 1998 e 2003. Ciênc Saúde Colet. 2006; 11 (4): 97586

9. Furtado MCDC, Braz JC, Pina JC, Mello DFD, Lima RAGD. Assessing the care of children under one year old in Primary Health Care. Rev Lat Am Enf. 2013; 21 (2): 554-61.

10. Cunha CLF, Silva RAD, Gama MEA, Costa GRC, Costa ASV, Tonia SR. O uso de serviços de atenção primária à saúde pela população infantil em um estado do nordeste brasileiro. Cad Saúde Colet. 2013; 21 (2): 115-20.

11. Cesar JA, Chrestani AD, Fantinel EJ, Gonçalves TS, Neumann NA. Saúde infantil em áreas pobres: resultados de um estudo de base populacional nos municípios de Caracol, Piauí, e Garrafão do Norte, Pará, Brasil. Cad Saúde Pública. 2009; 25 (4): 809-18.

12. Brasil. Ministério da Saúde. Secretaria de Atenção à Saúde Departamento de Atenção Básica. Saúde da criança: crescimento e desenvolvimento. Brasília, DF; 2012.

13. Piccini RX, Facchini LA, Tomasi E, Thumé E, da Silveira, DS, Siqueira FV, Rodrigues MA, Paniz VV, Teixeira VA. 
Efetividade da atenção pré-natal e de puericultura em unidades básicas de saúde do Sul e do Nordeste do Brasil. Rev Bras Saúde Matern Infant. 2007; 7 (1): 75-82.

14. Macinko J, Harris MJ. Brazil's Family Health StrategyDelivering Community-Based Primary Care in a Universal Health System. New England J Med. 2015; 372 (23): 2177 81.

15. Facchini LA, Piccini RX, Tomasi E, Thumé E, Silveira DS, Siqueira FV, Rodrigues MA. Desempenho do PSF no Sul e no Nordeste do Brasil: avaliação institucional e epidemiológica da Atenção Básica à Saúde. Ciênc Saúde Colet. 2006; 11 (3): 669-81

16. Facchini LA, Nunes BP, Motta JVS, Tomasi E, Silva, SM, Thumé E, et al. Insegurança alimentar no Nordeste e Sul do Brasil: magnitude, fatores associados e padrões de renda per capita para redução das iniqüidades. Cad Saúde Pública. 2014; 30 (1): 161-74.

17. Kamakura W. Socioeconomic stratification criteria and classification tools in Brazil. Rev Adm Empres. 2016; 56 (1): $55-70$

18. Caldeira AP, Oliveira RMD, Rodrigues OA. Qualidade da assistência materno-infantil em diferentes modelos de Atenção Primária. Ciênc Saúde Colet. 2010; 15 (Supl. 2): 3139-47.

19. Reis RS, Coimbra LC, da Silva AAM, dos Santos AM, Lamy ZC, Ribeiro SVO, da Silva RA. Acesso e utilização dos serviços na Estratégia Saúde da Família na perspectiva dos gestores, profissionais e usuários. Ciênc Saúde Colet. 2013; 18 (11): 3321-31.

20. Alves MGM, Casotti E, Oliveira LGD, Machado MTC, Almeida PF, Corvino MPF, Marin J, Flauzino RF, Montenegro LAA. Fatores condicionantes para o acesso às equipes da Estratégia Saúde da Família no Brasil. Saúde Debate. 2014; 38 (Especial): 34-51.

21. Tomasi E, Nunes BP, Müller RDM, Thumé E, SilveiraSD, Siqueira FV, Duro SMS, Saes MO, Dilélio AS, Reis MBD Facchini LA.Perfil de utilização de serviços de saúde por crianças de zona urbana no Brasil: estudo transversal de base nacional. Rev Bras Saúde Matern Infant. 2015; 15 (1): $81-90$.
22. Dias da Costa JS, Gigante DP, Horta BL, Barros FC, Victora CG. Utilização de serviços de saúde por adultos da coorte de nascimentos de 1982 a 2004-5, Pelotas, RS. Rev Saúde Pública. 2008; 42 (Supl. 2): 51-9.

23. Dias da Costa JS, Cesar JA, Weber AP, da Silva Garcez A, Dalla Nora CR, Rower, HB, Kolling V. Características das crianças menores de cinco anos atendidas em serviços de atenção básica em dois municípios do nordeste brasileiro. Rev Bras Saúde Matern Infant. 2015: 15 (1): 33-46.

24. Marmot M, Allen J, Bell R, Bloomer E, Goldblatt P. WHO European review of social determinants of health and the health divide. Lancet. 2012; 380 (9846): 1011-29.

25. Buss PM, Pellegrini Filho A. Iniquidades em saúde no Brasil, nossa mais grave doença: comentários sobre o documento de referência e os trabalhos da Comissão Nacional sobre Determinantes Sociais da Saúde. Cad Saúde Pública. 2006; 22 (9): 2005-8.

26. Quansah E, Ohene LA, Norman L, Mireku MO, Karikari TK. Social Factors Influencing Child Health in Ghana. PloS One. 2016; 11 (1): 2-20.

27. Langellier BA, Chen J, Vargas-Bustamante A, Inkelas M, Ortega AN. Understanding health-care access and utilization disparities among Latino children in the United States. J Child Health Care. 2016; 20 (2): 133-44.

28. Travassos C, Castro MSM. Determinantes e Desigualdades Sociais no Acesso e na Utilização dos Serviços de Saúde. In: Giovanella L, Sarah E, Lobato LVC, Noronha JC, Carvalho AI. Políticas e Sistemas de Saúde no Brasil. Parte I: Proteção Social, Políticas e Determinantes de Saúde. Fiocruz; 2012. p. 183-206

29. Jacobs B, Ir P, Bigdeli M, Annear PL, Van Damme W. Addressing access barriers to health services: an analytical framework for selecting appropriate interventions in lowincome Asian countries. Health Policy Plan. 2012; 27 (4): 288-300.

Received on January 11, 2017

Final version presented on May 24, 2017

Approved on June 20, 2017 